\title{
DYNAMIC CONDITIONAL CORRELATIONS FOR ASYMMETRIC PROCESSES
}

\author{
Manabu Asai* and Michael McAleer** *** **** *****
}

\begin{abstract}
The paper develops a new Dynamic Conditional Correlation (DCC) model, namely the Wishart DCC (wDCC) model. The paper applies the wDCC approach to the exponential GARCH (EGARCH) and GJR models to propose asymmetric DCC models. We use the standardized multivariate $t$-distribution to accommodate heavytailed errors. The paper presents an empirical example using the trivariate data of the Nikkei 225, Hang Seng and Straits Times Indices for estimating and forecasting the wDCC-EGARCH and wDCC-GJR models, and compares the performance with the asymmetric BEKK model. The empirical results show that AIC and BIC favour the wDCC-EGARCH model to the wDCC-GJR, asymmetric BEKK and alternative conventional DCC models. Moreover, the empirical results indicate that the wDCCEGARCH- $t$ model produces reasonable VaR threshold forecasts, which are very close to the nominal $1 \%$ to $3 \%$ values.
\end{abstract}

Key words and phrases: Asymmetric BEKK, dynamic conditional correlations, EGARCH, GJR, heavy-tailed errors, Wishart process.

\section{Introduction}

The class of multivariate Generalized Autoregressive Conditional Heteroskedasticity (GARCH) models has been used to model the co-movements of volatilities in financial assets. The various model specifications can be categorized as follows: (i) diagonal GARCH model of Bollerslev et al. (1998) and Ding and Engle (2001); (ii) BEKK (Baba, Engle, Kraft and Kroner) model of Engle and Kroner (1995), which models the conditional covariances directly; (iii) constant conditional correlation (CCC) model of Bollerslev (1990), VARMAGARCH model of Ling and McAleer (2003), and VARMA-AGARCH model of McAleer et al. (2009); (iv) Engle's (2002) dynamic conditional correlation (DCC) model, Tse and Tsui's (2002) varying conditional correlation (VCC) model, and Bauwens et al.'s (2006) generalized DCC model, and McAleer et al.'s (2008) Generalized Autoregressive conditional correlation (GARCC) model, which relax the assumption of constant conditional correlations and model the dynamic conditional correlations and covariances; (v) generalized orthogonal GARCH model of van der Weide (2002); and (vi) the matrix-exponential GARCH model of Kawakatsu (2006). For further details of these models, see the review papers of McAleer (2005) and Bauwens et al. (2006).

\footnotetext{
Received September 13, 2010. Revised February 7, 2011. Accepted April 7, 2011.

*Faculty of Economics, Soka University, 1-236 Tangi-machi, Hachioji, Tokyo, Japan.

**Econometric Institute, Erasmus School of Economics, Erasmus University Rotterdam.

$* * *$ Tinbergen Institute, The Netherlands.

****Institute of Economic Research, Kyoto University, Japan.

*****Department of Quantitative Economics, Complutense University of Madrid, Spain.
} 
For multivariate GARCH models, the primary concerns are the positivedefiniteness of the conditional covariance matrices and the large numbers of parameters. Regarding the latter issue, the number of parameters increases with the square of the dimension. One of the primary advantages of the DCC, VCC and GARCC models is that they reduce drastically the number of parameters in the time-varying structures of the conditional correlation and covariance matrices.

In the framework of univariate models, the asymmetric GARCH approach is typically modelled by using either the exponential GARCH (EGARCH) model of Nelson (1991) or the GJR (alternatively, the threshold GARCH) model of Glosten et al. (1992), whereby positive and negative shocks or equal magnitude have different effects on conditional volatility. The GJR model uses a threshold indicator function to describe the asymmetric effects. On the other hand, one of the appealing features of the EGARCH model is that it is a discrete time approximation to the continuous time asymmetric stochastic volatility model, as shown in Nelson (1990). Although Deb (1996) showed that the absolute value function in the EGARCH model is known to lead to bias in finite samples, the problem can be avoided by either of the following two approaches: (i) approximate the absolute value function by the rectangular hyperbola rotated counterclockwise by 45 degrees; or (ii) employ two step estimation for the conditional mean and conditional variance components (see Hentschel (1995) for further details).

For multivariate models, Kroner and Ng (1998) proposed the asymmetric BEKK model, while McAleer et al. (2009) suggested the asymmetric VARMAGARCH (or VARMA-AGARCH) model as a multivariate extension of the GJR model. Both of these models are multivariate generalizations of the univariate GJR model as they are based on threshold effects. Although the former is very flexible due to the BEKK specification, it suffers from the traditional large number of parameters associated with the BEKK specification. The latter model is an extension of the VARMA-GARCH model, and hence assumes constant conditional correlations. Recently, Kawakatsu (2006) suggested the matrixexponential GARCH model, which is a multivariate extension of the EGARCH model.

The purpose of this paper is to develop alternative specifications within the DCC class, based on the Wishart distribution. We employ the new DCC specification to propose two asymmetric DCC GARCH models, which are based on the EGARCH and GJR models, respectively. For the heavy-tails associated with financial returns, the standardized multivariate $t$-distribution is used. As a benchmark, we will use the asymmetric BEKK model.

In the remainder of the paper, Section 2 develops the new DCC models. Section 3 applies the theoretical results to suggest the asymmetric DCC class based on the GJR and EGARCH models. Section 4 explains the maximum likelihood estimation of the new DCC models, and investigates finite sample properties of the estimator. Section 5 presents an empirical example using the trivariate data of the Nikkei 225 Index, Hang Seng Index and Straits Times Index, and examines estimation of the parameters and forecasts of the VaR thresholds, 
based on the new class of models.

\section{Alternative DCC models}

\subsection{Background}

Let the returns on $m(\geq 2)$ financial assets be given by

$$
y_{t}=\mu_{t}+\varepsilon_{t},
$$

where $y_{t}=\left(y_{1 t}, \ldots, y_{m t}\right)^{\prime}, \varepsilon_{t}=\left(\varepsilon_{1 t}, \ldots, \varepsilon_{m t}\right)^{\prime}, \mu_{t}=\left(\mu_{1 t}, \ldots, \mu_{m t}\right)^{\prime}=E\left(y_{t}\right.$ $\left.\Im_{t-1}\right)$, and $\Im_{t}$ is the past information available at time $t$. It is assumed that

$$
\begin{aligned}
& \varepsilon_{t}=D_{t} \eta_{t}, \\
& \eta \mid \Gamma_{t} \sim \operatorname{iid}\left(0, \Gamma_{t}\right),
\end{aligned}
$$

where $\Gamma_{t}$ denotes the time-varying conditional correlation matrix, $D_{t}=$ $\left[\operatorname{diag}\left\{h_{t}\right\}\right]^{1 / 2}, h_{t}=\left(h_{1 t}, \ldots, h_{m t}\right)^{\prime}, \operatorname{diag}\{x\}$ for any vector $x$ denotes a diagonal matrix with $x$ along the diagonal, and $h_{i t}$ is the conditional variance for each asset.

It then follows that the conditional covariance matrix is given by

$$
Q_{t}=V\left(y_{t} \mid \Im_{t-1}\right)=E\left(\varepsilon_{t} \varepsilon_{t}^{\prime} \mid \Im_{t-1}\right)=D_{t} \Gamma_{t} D_{t} .
$$

While some authors, including Bollerslev et al. (1988) and Engle and Kroner (1995), have developed multivariate GARCH specifications in order to model, Engle (2002) concentrated on modelling $\Gamma_{t}$, the matrix of dynamic conditional correlations.

By using the Hadamard product, Ding and Engle (2001) provided a new representation of the diagonal GARCH model that was developed by Bollerslev et al. (1988). The simplest diagonal GARCH model is given as follows:

$$
Q_{t}=\Omega+\Psi_{1} \circ \varepsilon_{t-1} \varepsilon_{t-1}^{\prime}+\Psi_{2} \circ Q_{t-1},
$$

where $\Omega, \Psi_{1}$ and $\Psi_{2}$ are assumed to be positive semi-definite matrices, and ' $\circ$ ' denotes the Hadamard product of two identically-sized matrices or vectors, which is computed simply by element-by-element multiplication. Ding and Engle (2001) argued that, if any one of $\Omega, \Psi_{1}$ and $\Psi_{2}$ is positive definite, then $Q_{t}$ will also be positive definite.

On the other hand, the CCC and DCC models assume that the conditional variance of each asset follows the GARCH process, that is:

$$
h_{t}=\omega+\alpha \circ \varepsilon_{t-1} \circ \varepsilon_{t-1}+\beta \circ h_{t-1},
$$

where $\omega=\left(\omega_{1}, \ldots, \omega_{m}\right)^{\prime}, \alpha=\left(\alpha_{1}, \ldots, \alpha_{m}\right)^{\prime}, \beta=\left(\beta_{1}, \ldots, \beta_{m}\right)^{\prime}$, If we specify $\Gamma_{t}=\Gamma$ for all $t$, then we have the CCC model, as proposed by Bollerslev (1990). Engle (2002) proposed the specification of $\Gamma_{t}$ as follows:

$$
\begin{aligned}
\Gamma_{t} & =C_{t}^{-1} P_{t} C_{t}^{-1}, \\
C_{t} & =\left[\operatorname{diag}\left\{\operatorname{vecd}\left(P_{t}\right)\right\}\right]^{1 / 2}, \\
P_{t} & =\left(\iota_{m} \iota_{m}^{\prime}-\Theta_{1}-\Theta_{2}\right) \circ P+\Theta_{1} \circ \eta_{t-1} \eta_{t-1}^{\prime}+\Theta_{2} \circ P_{t-1},
\end{aligned}
$$


where $\iota_{m}$ is the $m \times 1$ unit vector, $P$ is a positive definite matrix, and 'vecd' creates a vector by stacking the diagonal elements of a matrix. As in the diagonal GARCH model, one of $\Theta_{1}, \Theta_{2}$ and $\left(\iota_{m} \iota_{m}^{\prime}-\Theta_{1}-\Theta_{2}\right)$ is assumed to be positive definite, and the remaining two can be positive definite or semi-definite.

Engle (2002) suggested a simpler model than in equation (2.9) that is based on scalar parameters, as follows:

$$
P_{t}=\left(1-\theta_{1}-\theta_{2}\right) P+\theta_{1} \eta_{t-1} \eta_{t-1}^{\prime}+\theta_{2} P_{t-1},
$$

where $\theta_{1}>0, \theta_{2}>0$ and $\theta_{1}+\theta_{2}<1$.

\subsection{Wishart approach}

In order to present the basic idea of the approach to be adopted in this paper, we will begin with a Wishart variate, $\Xi_{t} \sim W_{m}(k, p)$, where $P$ may be the constant part of the time-varying correlation matrix, as given in equation (2.9). Now consider the following process:

$$
P_{t}=\frac{1}{k} P_{t-1}^{d / 2} \Xi_{t}^{1-d} P_{t-1}^{d / 2},
$$

where $k>1$ and $|d|<1$. The last condition is required for stationarity. Taking the log-determinant of both sides of equation (2.11) gives

$$
\log \left|P_{t}\right|=-\log k+d \log \left|P_{t-1}\right|+(1-d) \log \left|\Xi_{t}\right|,
$$

so that $\log \left|P_{t}\right|$ follows an $\mathrm{AR}(1)$ process. Clearly, $\log \left|P_{t}\right|$ is the weighted average of $\log \left|P_{t-1}\right|$ and $\left|k^{-1} \Xi_{t}\right|$. As the mean of $\Xi_{t}$ is $k P$, this representation provides the motivation for the approach to be adopted in the paper.

It should be noted that the model in equation (2.11) is different from the Wishart Inverse Covariance (WIC) model of Asai and McAleer (2009) in the sense that $1-d$ is the exponent of $\Xi_{t}$, so that $P_{t}$ in equation (2.11) does not have the Wishart distribution, unlike the WIC model. However, the weighted average of $\log \left|P_{t}\right|$ arises from the presence of $1-d$.

Based on the above structure, we now propose a new DCC model. If $\eta_{t}$ has a normal distribution, then $\eta_{t} \eta_{t}^{\prime} \mid P_{t} \sim W_{m}\left(1, P_{t}\right)$. However, as $\eta_{t} \eta_{t}^{\prime}$ is positive semi-definite, it cannot be a proxy for $\Xi_{t+1}$. Instead, $\eta_{t} \eta_{t}^{\prime}+(k-1) P$ is used in order to derive

$$
P_{t}=\frac{1}{k} P_{t-1}^{d / 2}\left[\eta_{t-1} \eta_{t-1}^{\prime}+(k-1) P\right]^{1-d} P_{t-1}^{d / 2},
$$

where $k>1$ and $|d|<1$. The number of parameters for the correlation structure is given by $0.5 m(m-1)+2$, which is the same as for the scalar DCC model. In order to distinguish this model from Engle's DCC, we will refer to it as the Wishart DCC (wDCC) model. 


\section{3. wDCC-EGARCH and wDCC-GJR models}

Using the DCC structure and the Wishart approach, we propose two new families of DCC models, namely the wDCC-EGARCH and wDCC-GJR models, that are based on equations (2.7), (2.8) and (2.12).

For the wDCC-EGARCH model, we assume that the conditional variance of each asset follows the EGARCH process, namely:

$$
\log h_{t}=\kappa+\phi \circ \log h_{t-1}+\gamma \circ \eta_{t-1}+\delta \circ\left|\eta_{t-1}\right|
$$

where $\kappa=\left(\kappa_{1}, \ldots, \kappa_{m}\right)^{\prime}, \phi=\left(\phi_{1}, \ldots, \phi_{m}\right)^{\prime}, \gamma=\left(\gamma_{1}, \ldots, \gamma_{m}\right)^{\prime}$ and $\delta=\left(\delta_{1}, \ldots\right.$, $\left.\delta_{m}\right)^{\prime}$. Depending on the values of the parameters, the EGARCH model can capture asymmetry and leverage, whereby negative shocks increase volatility and positive shocks decrease volatility.

In the wDCC-GJR model, the conditional variance of each asset follows the GJR process, namely:

$$
h_{t}=\omega+\left(\alpha^{+} \circ\left\{\iota_{m}-d_{t-1}^{-}\right\}+\alpha^{-} \circ d_{t-1}^{-}\right) \circ \varepsilon_{t-1} \circ \varepsilon_{t-1}+\beta \circ h_{t-1},
$$

where $\alpha^{+}=\left(\alpha_{1}^{+}, \ldots, \alpha_{m}^{+}\right)^{\prime}$ and $\alpha^{-}=\left(\alpha_{1}^{-}, \ldots, \alpha_{m}^{-}\right)^{\prime}$ are $m$-vector of parameters. The vector $d_{t}^{-}=\left(d_{1 t}^{-}, \ldots, d_{m t}^{-}\right)^{\prime}$ denotes a set of indicator variables, and $d_{i t}^{-}$takes the value of one if $\varepsilon_{i t}<0$, and zero otherwise.

With respect to the conditional distribution for $\varepsilon_{t}$, we consider the multivariate normal and the multivariate $t$-distribution. The former specification is also used for the quasi-maximum likelihood estimation, which will be discussed in the next section. For convenience we denote wDCC-EGARCH-n and wDCC-EGARCH- $t$ corresponding to the normal distribution and $t$-distributions, respectively.

In the reminder of the section, we will give discussion regarding alternative specifications of the asymmetric multivariate conditional volatility models.

Recently, Kawakatsu (2006) developed the matrix exponential GARCH model, while Asai et al. (2006) proposed the matrix exponential SV model. Compared with the DCC-EGARCH model that is proposed here, the other two approaches have certain drawbacks. First, these two models are based on the unconditional (or unstandardized) shocks, $\varepsilon_{t}$, instead of the standardized shocks, $\eta_{t}$, in order to describe the leverage and size effects. In this sense, the matrix exponential GARCH model is not a direct extension of the EGARCH model. Second, the matrix exponential GARCH and matrix exponential SV models suffer from having a large number of parameters, as in the case of the BEKK model. Although the respective authors considered alternative ways of reducing the numbers of parameters, they still exceed those of the scalar DCC-EGARCH model. Third, the interpretation of the parameters is not straightforward as matrix exponentiation is not element-by-element exponentiation. As explained in Kawakatsu (2006), it requires additional computations in order to derive the relations between the $(i, j)$ element of the covariance matrix and the $(k, l)$ element of the matrix-logarithmic process. 
It should also be noted that the matrix exponential SV model of Asai et al. (2006) should perhaps be reconsidered since it is a multivariate SV model. In this regard, the superiority of univariate SV models over the GARCH and EGARCH models have frequently been shown in the volatility literature (see, for example, the review by McAleer (2005)).

Alternative asymmetric GARCH models are multivariate extensions of the GJR model. Kroner and Ng (1998) developed the asymmetric BEKK model, while McAleer et al. (2009) proposed the asymmetric VARMA-GARCH (or VARMA-AGARCH) model. The latter model assumes that the conditional correlations are constant. The asymmetric BEKK model is given by

$$
Q_{t}=K K^{\prime}+A \varepsilon_{t-1} \varepsilon_{t-1}^{\prime} A^{\prime}+B Q_{t-1} B^{\prime}+C \varepsilon_{t-1}^{*} \varepsilon_{t-1}^{* \prime} C^{\prime}
$$

where $K$ is the lower triangular matrix, $A, B$ and $C$ are square matrices, and $\varepsilon_{t}^{*}=\min \left(\varepsilon_{t}, 0\right)=(1 / 2)\left\{\varepsilon_{t}-\left|\varepsilon_{t}\right|\right\}$.

In addition to these models, we suggest the asymmetric diagonal GARCH model, which is given by

$$
Q_{t}=\Omega+\left(A+C \circ d_{t-1}^{-} d_{t-1}^{-\prime}\right) \circ \varepsilon_{t-1} \varepsilon_{t-1}^{\prime}+B \circ Q_{t-1}
$$

where the vector $d_{t}^{-}=\left(d_{1 t}^{-}, \ldots, d_{m t}^{-}\right)^{\prime}$ denotes a set of indicator variables, and $d_{i t}^{-}$takes the value of one if $\varepsilon_{i t}$ is negative, and zero otherwise. This model is a multivariate extension of the GJR model. Like the relation between the BEKK and diagonal GARCH models, diagonal specifications such that $A=\operatorname{diag}\{a\}$, $B=\operatorname{diag}\{b\}$ and $C=\operatorname{diag}\{c\}$ in (refeqn:abekk) yield

$$
Q_{t}=K K^{\prime}+\left(a a^{\prime}\right) \circ \varepsilon_{t-1} \varepsilon_{t-1}^{\prime}+\left(b b^{\prime}\right) \circ Q_{t-1} B^{\prime}+\left(c c^{\prime}\right) \circ \varepsilon_{t-1}^{*} \varepsilon_{t-1}^{* \prime},
$$

which is also a vector diagonal specification.

\section{Estimation}

We now consider estimation of the wDCC-GARCH, wDCC-EGARCH and wDCC-GJR models. Assuming normality of the conditional distribution of the standardized residuals, we can estimate the parameters by the maximum likelihood (ML) method for the DCC class of models. The conditional log-likelihood function is given by

$$
\begin{aligned}
L & =\sum_{t=1}^{T} l_{t}, \\
l_{t} & =-\frac{m}{2} \log (2 \pi)-\log \left|D_{t}\right|-\frac{1}{2} \log \left|\Gamma_{t}\right|-\frac{1}{2} \varepsilon_{t}^{\prime} D_{t}^{-1} \Gamma_{t}^{-1} D_{t}^{-1} \varepsilon_{t} .
\end{aligned}
$$

If the assumption of normality does not hold for the standardized residuals, the procedure is defined as the quasi-maximum likelihood estimator (QMLE). For more efficient estimators using adaptive methods, see Ling and McAleer (2003). 
As an alternative to the Gaussian assumption, we consider the standardized multivariate $t$-distribution for the conditional distribution. In this case, the contribution to the log-likelihood function from observation $t$ is

$$
l_{t}=c(\nu, m)-\log \left|D_{t}\right|-\frac{1}{2} \log \left|\Gamma_{t}\right|-\frac{\nu+m}{2} \log \left(1+\frac{\varepsilon_{t}^{\prime} D_{t}^{-1} \Gamma_{t}^{-1} D_{t}^{-1} \varepsilon_{t}}{\nu-2}\right),
$$

where

$$
c(\nu, m)=\log \Gamma\left(\frac{\nu+m}{2}\right)-\log \Gamma\left(\frac{\nu}{2}\right)-\frac{m}{2} \log \pi-\frac{m}{2} \log (\nu-2),
$$

$\Gamma(x)$ is the complete gamma function, and $\nu$ is the degrees of freedom parameter. For the specification, $\varepsilon_{t}$ has the multivariate $t$-distribution with mean zero and variance $Q_{t}$. The multivariate $t$-distribution has a convenient property for the portfolio analysis with heavy-tailed distribution. If we consider a portfolio $\varepsilon_{p, t}=$ $w^{\prime} \varepsilon_{t}$ with weight vector $w, \varepsilon_{p, t}$ has the $t$-distribution with mean zero and variance $w^{\prime} Q_{t} w$.

As in Engle (2002), we may conduct two-step (Q)ML estimation for the Gaussian conditional distribution. The first step is to estimate conditional volatility model for each equation, while the second step is to estimate the wDCC model using the reminder part of the (quasi-)log-likelihood function. See also Song et al. (2005) for the theoretical results for the approach. In the reminder of the paper, we only consider one-step estimation, as we mainly consider the multivariate $t$-distribution.

Now we investigate the finite sample properties of the ML estimators through Monte Carlo simulations for the wDCC models. As our concern is on the parameters for the dynamic correlation, we employ a simple bivariate wDCC-GARCH-n model.

We consider two data-generating processes (DGP). We specify the parameters of the dynamic correlations in (2.12) as

$$
\left(P_{12}, k, d\right)= \begin{cases}(0.3,1.5,0.9) & \text { for DGP1 } \\ (-0.3,2,0.95) & \text { for DGP2 }\end{cases}
$$

and set the parameters of the conditional variances in (refeqn:garch) as $\left(\omega_{1}, \alpha_{1}, \beta_{1}\right)=(0.05,0.15,0.8)$ and $\left(\omega_{2}, \alpha_{2}, \beta_{2}\right)=(0.02,0.1,0.88)$. Here, $P_{12}$ is the $(1,2)$-element of $P$. With respect to the dynamic correlations, the degree of persistence is given by $d$. Hence, DGP2 shows higher persistence in the correlation dynamics. The parameter of the unconditional correlation, $P_{12}$, is 0.3 for DGP1, while that is -0.3 for DGP2. The Wishart distribution has 1.5 degrees of freedom for DGP1, but 2 for DGP2. Turning to the conditional variance, the second component indicates higher persistence in the variance process than the first component does. These two sets are common between DGP1 and DGP2. We consider a sample size of $T=500$ with 1000 replications.

Table 1 shows the sample means, standard deviations and root mean squared errors (RMSE) of the ML estimators. Small biases and standard deviations are 
Table 1. Finite sample performance of the ML estimator for the wDCC-GARCH-n model with $T=500$.

\begin{tabular}{c|cccc}
\hline Parameters & \multicolumn{2}{|c}{ DGP1 } & \multicolumn{2}{c}{ DGP2 } \\
& $i=1$ & $i=2$ & $i=1$ & $i=2$ \\
\hline$\omega_{i}$ & 0.0057 & 0.0009 & 0.0055 & 0.0008 \\
& $(0.0046)$ & $(0.0008)$ & $(0.0044)$ & $(0.0007)$ \\
& {$[0.0446]$} & {$[0.0191]$} & {$[0.0447]$} & {$[0.0192]$} \\
$\alpha_{i}$ & 0.1315 & 0.1097 & 0.1320 & 0.1107 \\
& $(0.0387)$ & $(0.0320)$ & $(0.0367)$ & $(0.0318)$ \\
& {$[0.0495]$} & {$[0.0334]$} & {$[0.0454]$} & {$[0.0335]$} \\
$\beta_{i}$ & 0.8075 & 0.8804 & 0.8084 & 0.8812 \\
& $(0.0483)$ & $(0.0323)$ & $(0.0481)$ & $(0.0331)$ \\
\multirow{5}{*}{$P_{2 i}$} & {$[0.0497]$} & {$[0.0323]$} & {$[0.0499]$} & {$[0.0332]$} \\
& 0.2904 & & -0.3014 & \\
& $(0.0313)$ & & $0.0693)$ & \\
$k$ & {$[0.0328]$} & & {$[0.0693]$} & \\
& 1.1576 & & 2.5709 & \\
& $(0.0144)$ & & $0.7023)$ & \\
& {$[0.0168]$} & & $0.7059]$ & \\
$d$ & 0.8971 & & 0.9368 & \\
& $(0.0185)$ & & $0.1042)$ & \\
& {$[0.0187]$} & & $0.1051]$ & \\
\hline
\end{tabular}

Note: Standard deviations are in parentheses and root mean squared errors are in brackets.

observed in almost all parameters. The RMSEs and the corresponding standard deviations are close, indicating that the biases are negligible. The only noticeable exception is the small downward bias in $\omega_{i}$. Such a bias is expected to disappear with a larger sample size, as shown by the Monte Carlo experiments with univariate and multivariate GARCH models (e.g., Lumsdaine (1995); Bollerslev and Wooldridge (1992)).

\section{Empirical results}

In this section, we examine the MLE of wDCC-EGARCH and wDCC-GJR models for three sets of empirical data, namely the Nikkei 225 Index (Nikkei), Hang Seng Index (Hang Seng), and Straits Times Index (Straits Times) returns. The sample period for the three data series is $1 / 4 / 1988$ to $7 / 17 / 2002$, giving $T=3773$ observations. Returns, $y_{i t}$, are defined as $100 \times\left\{\log P_{i t}-\log P_{i, t-1}\right\}$, where $P_{i t}$ is the closing price on day $t$ for stock $i$. We use the filtered data, $\varepsilon_{i t}=y_{i t}-\mu_{i t}$, based on the threshold AR(1) model.

Table 2 shows AIC and BIC for several kinds of trivariate DCC models along with the asymmetric BEKK model. With respect to the dynamic conditional correlation, we compare the wDCC model with the DCC model of Engle (2002). For the conditional volatility and conditional distribution, we consider the GARCH-n, GARCH- $t$, GJR- $t$, and EGARCH- $t$ specifications. The AIC and 
Table 2. AIC and BIC for DCC, wDCC and BEKK models.

\begin{tabular}{c|cccc}
\hline Volatility & \multicolumn{2}{|c}{$\begin{array}{c}\text { Engle's (2002) } \\
\text { DCC Model }\end{array}$} & \multicolumn{2}{c}{ Wishart DCC Model } \\
& AIC & BIC & AIC & BIC \\
\hline GARCH-n & 35727.1 & 35814.4 & 35738.1 & 35825.3 \\
GARCH- $t$ & 35653.9 & 35759.8 & 34734.3 & 34827.8 \\
GJR- $t$ & 35548.8 & 35654.8 & 34629.0 & 34741.2 \\
EGARCH- $t$ & 35568.2 & 35674.2 & $34598.0^{*}$ & $34710.2^{*}$ \\
Benchmark Model & & & & \\
AD-BEKK- $t$ & 34638.4 & 34738.1 & & \\
\hline
\end{tabular}

Note: '*' denotes the minimum AIC (BIC) among the alternative models.

Table 3. Estimates of trivariate wDCC-GARCH-n model.

\begin{tabular}{c|ccc}
\hline Parameters & Nikkei & Hang Seng & Straits Times \\
\hline$\omega_{i}$ & 0.0209 & 0.0656 & 0.0785 \\
& $(0.0039)$ & $(0.0089)$ & $(0.0082)$ \\
$\alpha_{i}$ & 0.0844 & 0.0971 & 0.1443 \\
& $(0.0078)$ & $(0.0083)$ & $(0.0114)$ \\
$\beta_{i}$ & 0.9091 & 0.8782 & 0.81288 \\
& $(0.0080)$ & $(0.0094)$ & $(0.0121)$ \\
$P_{2 i}$ & 0.3247 & 1 & \\
& $(0.0212)$ & & \\
$P_{3 i}$ & 0.3010 & 0.4644 & \\
& $(0.0212)$ & $(0.0188)$ & \\
$k$ & 1.6682 & & \\
& $(0.1839)$ & & \\
$d$ & 0.9036 & & \\
LogLike & $(0.0210)$ & & \\
AIC & 35855.0 & & \\
BIC & 35825.3 & & \\
\hline
\end{tabular}

Note: Standard errors are in parentheses. The structure of the DCC model in this paper is given in equation (2.12).

BIC criteria favour the new wDCC to Engle's (2002) DCC model, implying that the wDCC model is competitive with the standard DCC version. The important point to be made is that the DCC specification of Engle (2002) is not the only approach for describing dynamic conditional correlations, and is certainly not the best approach empirically.

Table 3 presents the ML estimates for the trivariate wDCC-GARCH-n model. For the GARCH parameters, the estimates of $\alpha_{i}+\beta_{i}$ are close to 0.98 for Nikkei and Hang Seng, while it is about 0.95 for Straits Times. The estimates of $P_{i j}$ 's are significant and greater than 0.30 . With respect to the persistence of the correlation structure, $\hat{d}$ is lower at 0.90 . The estimate of $k$ is 1.6 , and is significantly 
Table 4. Estimates of trivariate wDCC-GARCH- $t$ model.

\begin{tabular}{c|ccc}
\hline Parameters & Nikkei & Hang Seng & Straits Times \\
\hline$\omega_{i}$ & 0.0171 & 0.0495 & 0.0614 \\
& $(0.0040)$ & $(0.0093)$ & $(0.0098)$ \\
$\alpha_{i}$ & 0.0783 & 0.0706 & 0.1323 \\
& $(0.0084)$ & $(0.0085)$ & $(0.0141)$ \\
$\beta_{i}$ & 0.9157 & 0.9033 & 0.8189 \\
& $(0.0085)$ & $(0.0110)$ & $(0.0177)$ \\
$P_{2 i}$ & 0.2659 & 1 & \\
& $(0.0255)$ & & 1 \\
$P_{3 i}$ & 0.2207 & 0.3671 & \\
& $(0.0261)$ & $(0.0251)$ & \\
$k$ & 1.6035 & & \\
& $(0.1735)$ & & \\
$d$ & 0.8951 & & \\
& $(0.0212)$ & & \\
$\nu$ & 6.5881 & & \\
LogLike & $(0.3557)$ & & \\
AIC & -17352.1 & & \\
BIC & 34734.3 & & \\
\hline
\end{tabular}

Note: Standard errors are in parentheses. The structure of the DCC model in this paper is given in equation (2.12).

greater than one, indicating that the positive definiteness is guaranteed.

Table 4 shows the ML results for the trivariate wDCC-GARCH- $t$ model. The estimate of $\nu$ is 6.59 , showing that the conditional distribution is far from a normal distribution. The likelihood ratio test rejects the null hypothesis of normality. Hence, we will employ the multivariate standardized $t$-distribution in the remainder of the paper. The estimates of correlations in $P$ for Table 4 are smaller than those in Table 3.

Table 5 gives the results for the trivariate wDCC-GJR-t model. The estimates of $\alpha_{i}^{+}$are significantly different from those of $\alpha_{i}^{-}$, indicating that there are asymmetric effects in the conditional volatilities. The AIC and BIC criteria also favour the wDCC-GJR- $t$ model relative to the wDCC-GARCH- $t$ model. The estimates of $P, d$ and $k$ are close to those of the wDCC-GARCH model, implying that the inclusion of asymmetric effects alters slightly the dynamic conditional correlations.

Table 6 gives the ML estimates of the trivariate wDCC-EGARCH- $t$ model. The estimates of $\delta_{i}$ are positive and significant, while those of $\gamma_{i}$ are negative and significant, which are typical for EGARCH specifications. The AIC and BIC criteria for the wDCC-EGARCH- $t$ model are smaller than those of the wDCCGARCH- $t$ model, while the estimates of $P, d$ and $k$ are close to those of the wDCC-GARCH- $t$ and wDCC-GJR- $t$ models. For the asymmetric models, the 
Table 5. Estimates of trivariate wDCC-GJR- $t$ model.

\begin{tabular}{|c|c|c|c|}
\hline Parameters & Nikkei & Hang Seng & Straits Times \\
\hline \multirow[t]{2}{*}{$\omega_{i}$} & 0.0215 & 0.0633 & 0.0650 \\
\hline & $(0.0043)$ & $(0.0105)$ & $(0.0099)$ \\
\hline \multirow[t]{2}{*}{$\alpha_{i}^{+}$} & 0.0400 & 0.0401 & 0.0958 \\
\hline & $(0.0099)$ & $(0.0082)$ & $(0.0144)$ \\
\hline \multirow[t]{2}{*}{$\alpha_{i}^{-}$} & 0.1359 & 0.1023 & 0.1749 \\
\hline & $(0.0142)$ & $(0.0128)$ & $(0.0195)$ \\
\hline \multirow[t]{2}{*}{$\beta_{i}$} & 0.9057 & 0.8956 & 0.8131 \\
\hline & $(0.0103)$ & $(0.0111)$ & $(0.0178)$ \\
\hline \multirow[t]{2}{*}{$P_{2 i}$} & 0.2700 & 1 & \\
\hline & $(0.0250)$ & & \\
\hline \multirow[t]{2}{*}{$P_{3 i}$} & 0.2246 & 0.3686 & 1 \\
\hline & $(0.0256)$ & $(0.0248)$ & \\
\hline \multirow[t]{2}{*}{$k$} & 1.6251 & & \\
\hline & $(0.1739)$ & & \\
\hline \multirow[t]{2}{*}{$d$} & 0.8860 & & \\
\hline & $(0.0222)$ & & \\
\hline \multirow[t]{2}{*}{$\nu$} & 6.9074 & & \\
\hline & $(0.3863)$ & & \\
\hline LogLike & -17296.5 & & \\
\hline AIC & 34629.0 & & \\
\hline $\mathrm{BIC}$ & 34741.2 & & \\
\hline
\end{tabular}

Note: Standard errors are in parentheses. The structure of the DCC model in this paper is given in equation (2.12).

AIC and BIC criteria both favour the wDCC-EGARCH- $t$ specification.

In order to compare the new asymmetric wDCC models, we also estimate the asymmetric BEKK model. We use the standardized multivariate $t$-distribution for the distribution of $\eta_{t}$. In order to reduce the number of parameters, we use diagonal specifications for $A, B$ and $C$, and refer to the asymmetric diagonal BEKK- $t$ model as AD-BEKK- $t$. It should be noted that the scalar BEKK models are not analyzed, as Engle (2002) showed the superiority of the DCC-GARCH model over the scalar BEKK model on the basis of Monte Carlo simulations.

The numbers of parameters for the DCC-GJR- $t$, DCC-EGARCH- $t$ and ADBEKK- $t$ are $.5\left(m^{2}+7 m\right)+3, .5\left(m^{2}+7 m\right)+3$ and $.5\left(m^{2}+7 m\right)+1$, respectively. For the number of parameters, the difference among the three models is 2 .

Table 7 shows the ML estimates for the AD-BEKK- $t$ model. The estimate of $\nu$ is 6.79 , showing the rejection of the normality assumption. The estimates of $c_{i i}$ are significant, indicating that the negative shock has a larger effect than a positive shock of similar magnitude. The AIC and BIC criteria for the ADBEKK- $t$ model are close to those of the wDCC-GJR- $t$ model. Among the wDCCGJR- $t$, wDCC-EGARCH- $t$ and AD-BEKK- $t$ models, the AIC and BIC criteria select the wDCC-EGARCH-t as the best empirically. 
Table 6. Estimates of trivariate wDCC-EGARCH- $t$ model.

\begin{tabular}{c|ccc}
\hline Parameters & Nikkei & Hang Seng & Straits Times \\
\hline$\kappa_{i}$ & -0.0828 & -0.0854 & -0.1641 \\
& $(0.0102)$ & $(0.0093)$ & $(0.0147)$ \\
$\phi_{i}$ & 0.9839 & 0.9771 & 0.9594 \\
& $(0.0026)$ & $(0.0040)$ & $(0.0068)$ \\
$\gamma_{i}$ & -0.0820 & -0.0415 & -0.0413 \\
& $(0.0086)$ & $(0.0085)$ & $(0.0098)$ \\
$\delta_{i}$ & 0.1234 & 0.1342 & 0.2226 \\
& $(0.0144)$ & $(0.0137)$ & $(0.0202)$ \\
$P_{2 i}$ & 0.2662 & 1 & \\
& $(0.0264)$ & & \\
$P_{3 i}$ & 0.2191 & 0.3614 & \\
& $(0.0269)$ & $(0.0261)$ & \\
$k$ & 1.5404 & & \\
& $(0.1624)$ & & \\
$d$ & 0.9013 & & \\
& $(0.0211)$ & & \\
$\nu$ & 6.8287 & & \\
LogLike & $(0.3915)$ & & \\
AIC & -17281.0 & & \\
BIC & 34598.0 & & \\
\hline & 34710.2 & & \\
\hline
\end{tabular}

Note: Standard errors are in parentheses. The structure of the DCC model in this paper is given in equation (2.12).

Next, we compare the out-of-sample forecasts for the Value-at-Risk (VaR) for the DCC, wDCC and asymmetric BEKK models. For this purpose, we define the portfolio of trivariate returns as $y_{p, t}=w^{\prime} y_{t}$, where $w$ is the vector of portfolio weights. Assuming that the conditional distribution is given by the multivariate $t$-distribution, we have $y_{p, t}=\mu_{p, t}+\varepsilon_{p, t}$, where $\mu_{p, t}=w^{\prime} \mu_{t}$ is the conditional mean and $\varepsilon_{p, t}$ has the $t$-distribution with mean zero and variance $h_{t}=w^{\prime} Q_{t} w$. See also Asai and McAleer (2008).

In this paper, we use the equally-weighted portfolio. Fixing the sample size in estimation to be 500, we re-estimate the model and forecast one-step-ahead VaR thresholds for the last 500 observations, where the 1 percent VaR threshold is given by $\hat{\mu}_{p, t \mid t-1}+t_{0.01}^{s}(\hat{\nu}) \times \sqrt{\hat{h}_{p, t \mid t-1}}$, where $\hat{\mu}_{p, t \mid t-1}$ and $\hat{h}_{p, t \mid t-1}$ are the onestep-ahead predictions of the mean and variance, respectively, and $t_{0.01}^{s}(\nu)$ is the 1 percentile of the standardized $t$-distribution with degrees-of-freedom given by $\nu$. Note that $\hat{\mu}_{p, t \mid t-1}$ is the same for all the models, and that this setting makes the effects of each volatility forecast more clear. We define the failure percentage as the ratio of the number of times that the portfolio return exceeds its forecast, i.e., $y_{p, t}<\hat{\mu}_{p, t \mid t-1}+t_{0.01}^{s}(\hat{\nu}) \times \sqrt{\hat{h}_{p, t \mid t-1}}$, divided by the number of out-of-sample forecasts. 
Table 7. Estimates of trivariate AD-BEKK- $t$ model.

\begin{tabular}{c|ccc}
\hline Parameters & Nikkei & Hang Seng & Straits Times \\
\hline$k_{1 i}$ & 0.1305 & 0 & 0 \\
& $(0.0128)$ & & \\
$k_{2 i}$ & 0.0380 & 0.0536 & 0 \\
& $(0.0173)$ & $(0.0193)$ & \\
$k_{3 i}$ & 0.2180 & 0.0594 & 0.2200 \\
& $(0.0198)$ & $(0.0136)$ & $(0.0150)$ \\
$a_{i i}$ & 0.0701 & 0.1986 & 0.2980 \\
& $(0.0219)$ & $(0.0163)$ & $(0.0186)$ \\
$b_{i i}$ & 0.9655 & 0.9549 & 0.9135 \\
& $(0.0034)$ & $(0.0046)$ & $(0.0076)$ \\
$c_{i i}$ & 0.3468 & 0.2236 & 0.2734 \\
& $(0.0189)$ & $(0.0270)$ & $(0.0294)$ \\
$\nu$ & 6.7878 & & \\
& $(0.3901)$ & & \\
LogLike & -17303.2 & & \\
AIC & 34638.4 & & \\
BIC & 34738.1 & & \\
\hline
\end{tabular}

Note: Standard errors are in parentheses.

Table 8. VaR forecasting performance.

\begin{tabular}{l|ccc}
\hline \multicolumn{1}{c|}{ Model } & $1 \%$ & $2 \%$ & $3 \%$ \\
\hline DCC-GJR- $t$ & 0.010 & 0.022 & 0.034 \\
wDCC-GARCH-n & 0.012 & 0.024 & 0.040 \\
wDCC-GARCH- $t$ & 0.012 & 0.028 & 0.036 \\
wDCC-GJR- $t$ & 0.010 & 0.022 & 0.042 \\
wDCC-EGARCH- $t$ & 0.008 & 0.018 & 0.030 \\
AD-BEKK- $t$ & 0.012 & 0.022 & 0.030 \\
\hline
\end{tabular}

Note: The entries show the \% violations of the VaR thresholds.

Table 8 gives the failure percentages for the VaR forecasts based on the DCCGJR- $t$, wDCC-GARCH-n, wDCC-GJR- $t$, wDCC-EGARCH- $t$ and AD-BEKK- $t$ models with respect to the true values for $1 \%-3 \%$. The tail behaviour of wDCCEGARCH- $t$ and AD-BEKK- $t$ two models is quite similar, although the wDCCEGARCH- $t$ produces slightly more conservative results.

\section{Concluding remarks}

In this paper, we proposed alternative Dynamic Conditional Correlation (DCC) models based on the Wishart distribution, in order to develop the new wDCC-EGARCH and wDCC-GJR models.

The standardized multivariate $t$-distribution was used to capture the wellknown heavy-tails associated with financial assets. An empirical example for 
the trivariate data of the Nikkei 225, Hang Seng and Straits Times Index returns showed that AIC and BIC favoured the wDCC-EGARCH- $t$ model to the wDCC-GJR- $t$, asymmetric BEKK- $t$ and alternative conventional DCC models. Moreover, the empirical results indicated that the wDCC-EGARCH- $t$ model produced reasonable $\mathrm{VaR}$ threshold forecasts, which are very close to the nominal $1 \%$ to $3 \%$ values.

\section{Acknowledgements}

The authors wish to thank the editor and two referees for insightful comments and suggestions and Yoshi Baba for helpful discussions. For financial support, the first author acknowledges the Japan Society for the Promotion of Science and the Australian Academy of Science, and the second author wishes to acknowledge the Australian Research Council, National Science Council, Taiwan, and the Japan Society for the Promotion of Science.

\section{REFERENCES}

Asai, M. and McAleer, M. (2008). A portfolio index GARCH model, Int. Jour. Forec., 24, $449-461$.

Asai, M. and McAleer, M. (2009). The structure of dynamic correlations in multivariate stochastic volatility models, J. Economet., 150, 182-192.

Asai, M., McAleer, M. and Yu, J. (2006). Multivariate stochastic volatility: A review, Economet. Rev., 25, 145-175.

Bauwens L., Laurent, S. and Rombouts, J. K. V. (2006). Multivariate GARCH models: A survey. J. Appl. Economet., 21, 79-109.

Bollerslev, T. (1990). Modelling the coherence in short-run nominal exchange rates: A multivariate generalized ARCH approach, Rev. Econ. Statist., 72, 498-505.

Bollerslev, T. and Wooldridge, J. M. (1992). Quasi-maximum likelihood estimation and inference in dynamic models with time-varying covariances, Economet. Rev., 11, 143-172.

Bollerslev, T., Engle, R. F. and Wooldridge, J. M. (1988). A capital asset pricing model with time varying covariances, J. Polit. Econ., 96, 116-131.

Chiu, T. Y. M., Leonard, T. and Tsui, K.-W. (1996). The matrix-logarithmic covariance model, J. Amer. Statist. Assoc., 91, 198-210.

Deb, P. (1996). Finite sample properties of the maximum likelihood estimator of EGARCH models, Economet. Rev., 15, 51-68.

Ding, Z. and Engle, R. F. (2001). Large scale conditional covariance matrix modeling, estimation and testing, Academia Economic Papers, 1, 83-106.

Engle, R. F. (2002). Dynamic conditional correlation: A simple class of multivariate generalized autoregressive conditional heteroskedasticity models, J. Bus. Econ. Statist., 20, 339-350.

Engle, R. F. and Kroner, K. F. (1995). Multivariate simultaneous generalized ARCH, Economet. Theory, 11, 122-150.

Glosten, L., Jagannathan, R. and Runkle, D. (1992). On the relation between the expected value and volatility of nominal excess returns on stocks, J. Fin., 46, 1779-1801.

Hentschel, L. (1995). All in the family: nesting symmetric and asymmetric GARCH models, J. Fin. Econ., 39, 71-104.

Kawakatsu, H. (2006). Matrix exponential GARCH, J. Economet., 134, 95-128.

Kroner, K. and Ng, V. (1998). Modeling asymmetric comovements of asset returns, Rev. Fin. Stud., 11, 817-844.

Ling, S. and McAleer, M. (2003). On adaptive estimation in nonstationary ARMA models with GARCH errors, Ann. Statist., 31, 642-674. 
Lumsdaine, R. L. (1995). Finite-sample properties of the maximum likelihood estimator in $\operatorname{GARCH}(1,1)$ and $\operatorname{IGARCH}(1,1)$ models: A Monte Carlo investigation, J. Bus. Econ. Statist., 13, 1-10.

McAleer, M. (2005). Automated inference and learning in modeling financial volatility, Economet. Theory, 21, 232-261.

McAleer, M., Chan, F., Hoti, S. and Lieberman, O. (2008). Generalized autoregressive conditional correlation, Economet. Theory, 24, 1554-1583.

McAleer, M., Hoti, S. and Chan, F. (2009). Structure and asymptotic theory for multivariate asymmetric conditional volatility, Economet. Rev., 28, 422-440.

Nelson, D. B. (1990). ARCH models as diffusion approximations, J. Economet., 45, 7-38.

Nelson, D. B. (1991). Conditional heteroskedasticity in asset returns: A new approach, Econometrica, 59, 347-370.

Song, P. X.-K., Fan, Y. and Kalbfleisch, J. D. (2005). Maximization by parts in likelihood inference, J. Amer. Statist. Assoc., 100, 1163-1167.

Tse, Y. K. and Tsui, A. K. C. (2002). A multivariate GARCH model with time-varying correlations, J. Bus. Econ. Statist., 20,351-362.

van der Weide, R. (2002). GO-GARCH: A multivariate generalized orthogonal GARCH model, J. Appl. Economet., 17, 549-564. 